\title{
réalisation de la fouille de l'usine de Brégnier-Cordon
}

\author{
work excavation of the Bregnier-Cordon power station
}

\author{
M. LLOPIS
}

Compagnie Nationale du Rhône*

\section{Résumé}

Après avoir situé l'emplacement des travaux de l'usine et précisé la stratigraphie du sol sur le site, l'exposé rappelle les conclusions résultant des essais de pompage.

L'exposé précise ensuite les caractéristiques géométriques de la fouille de l'usine, ainsi que l'ensemble du dispositif de rabattement mis en place avec les contraintes de la maintenance de ce dispositif pendant toute la durée des travaux d'exécution.

Le volume réel de rabattement et l'évolution des débits correspondants sont donnés en fin d'exposé.

\section{Abstract}

After the works of the power station have been located and the stratigraphy of the soil on the spot has been specified, the report recalls to memory the conclusions resulting from the pumping tests.

Then, the report explains the special features of the excavation of the power station and of the pumping device which needed an uninterrupted maintenance during the execution of the works.

The actual lowering volume and the corresponding discharges variations are listed at the end of the report. 


\section{SITUATION DE LA CHUTE DE BRÉGNIER-CORDON}

La chute de Brégnier-Cordon s'inscrit dans le cadre de l'aménagement hydro-électrique du Haut-Rhône dont elle constitue la, troisième chute réalisée à l'aval de Seyssel sur l'ensemble des cinq prévues. Situé entre Belley et le pont d'Evieu à l'aval, laménagement de la chute de Brégnier-Cordon intéresse une longueur du Rhône de 28 km environ; il comprend, de l'amont vers l'aval :

- une retenue de $12 \mathrm{~km}$;

- un barrage de retenue situé en rive gauche du Rhône sur la commune de Champagneux et complété par une petite usine hydro-électrique :

- un canal d'amenée de $5 \mathrm{~km}$ de longueur qui s'écarte de la plaine du Rhône en empruntant la dépression occupée par le lac de Pluvis et en passant au sud du village de Brégnier-Cordon;

- une usine hydro-électrique, située en rive droite du Rhône, dans le lit majeur, sur la commune de BrégnierCordon; cette usine comporte deux groupes bulbes capables d'une production annuelle moyenne de $360 \mathrm{GWh}$ (360 millions de kWh), avec une chute de hauteur maximale $14,3 \mathrm{~m}$ environ et un débit d'équipement de $700 \mathrm{~m}^{3} / \mathrm{s}$;

- un canal de fuite de $3 \mathrm{~km}$ de longueur creusé dans les alluvions fluviales de la plaine du Rhône et dans les silts sous-jacents.

\section{GÉOLOGIE ET STRATIGRAPHIE DU SOL SUR LE SITE DE L'USINE}

\subsection{Stratigraphie du terrain (fig. 1)}

Les sondages de reconnaissance réalisés sur le site de l'usine au cours de deux campagnes successives, les analyses effectuées au laboratoire sur échantillons et la synthèse des caractéristiques des sols ont permis d'aboutir à la stratification suivante du terrain.

La cote du terrain naturel, pratiquement horizontal dans toute la zone, est voisine du niveau N.G.F. $(207,00)$ et celle de la nappe de $(206,00)$ environ. Sous une faible épaisseur de terre végétale et de limon argilo-sableux (2,00 m environ), on rencontre une couche de graviers sableux d'épaisseur moyenne $8 \mathrm{~m}$ à $9 \mathrm{~m}$, puis une couche de sables fins silteux pratiquement non plastiques de caractéristiques mécaniques élevées et peu compressibles, très peu perméables (perméabilité de l'ordre de $8,10^{-6} \mathrm{~m} / \mathrm{s}$ ) avec intercalations plus marneuses et, vers $42 \mathrm{~m}$ environ au-dessous du terrain naturel (cote N.G.F. 165,00 environ), un horizon de 2 à $3 \mathrm{~m}$ d'épaisseur constitué de silts plus fins et moins perméables.

Par ailleurs, les essais réalisés sur les échantillons prélevés dans les sondages lors de la dernière campagne ont permis de mettre en évidence la forte anisotropie du sol de fondation. Enfin, un sondage, arrêté dans les sables silteux à $100 \mathrm{~m}$ de profondeur, n'a pas permis de reconnaître un éventuel fond étanche sous les sables silteux.

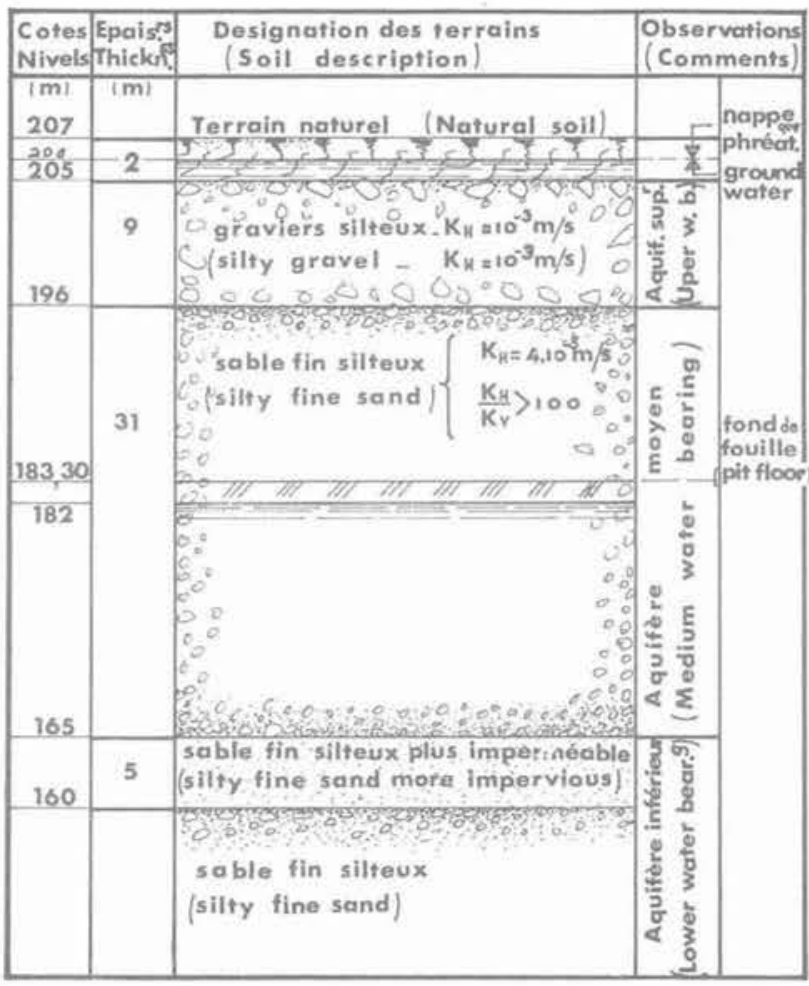

\section{Fig. 1. - Coupe schématique du terrain sur le site de l'usine \\ (soil schematic profile on the location of the power station)}

\subsection{Essais de pompage}

Les essais de pompage avaient pour objet :

- de tester in situ la perméabilité et l'anisotropie des sables silteux dans lesquels seraient réalisées les fouilles de l'usine;

- de déterminer la capacité de rabattement des puits envisagés, avec leurs caractéristiques dimensionnelles;

- de tester les techniques de réalisation des puits de rabattement.

Préalablement à la réalisation des essais de pompage, il est apparu nécessaire d'effectuer une étude de la schématisation de ces essais sur modèles analogique électrique bidimensionnel et mathématique tridimensionnel. Cette étude tenait compte d'un ensemble de dispositions pratiques liées à la nature des travaux à réaliser: dimensions de la fouille du batardeau de protection du chantier, écran périphérique isolant l'aquifère perméable sur la hauteur des graviers, horizons plus marneux dans les silts qu'elle assimilait à un milieu homogène et anisotrope du point de vue des perméabilités avec un rapport d'anisotropie $\mathrm{a} \frac{\mathrm{k}_{\mathrm{H}}}{\mathrm{k}_{\mathrm{v}}}$. Après étude de la variation du potentiel hydraulique en fonction des rapports d'anisotropie (variant de 1 à 400) en des points distants de $5,10,20,50$ et $100 \mathrm{~m}$ du puits, le schéma de principe à adopter pour l'essai de pompage était défini. 
Les essais de pompage ont pu être ainsi réalisés in situ sur des puits de gros diamètre exécutés suivant deux techniques : le vibro-fonçage et le forage en rotation à la boue autodestructible du type *REVERT».

De ces essais de pompage, il ressortait que :

- le forage des puits par vibro-fonçage était à proscrire ; seul le forage en rotation à la boue autodestructible donnait satisfaction: le forage à l'eau claire s'était révélé difficile, sinon impossible :

- la perméabilité horizontale dans les sables silteux était, en moyenne, de l'ordre de $\mathrm{k}_{H}=4,10^{-5} \mathrm{~m} / \mathrm{s}$;

- le rapport d'anisotropie : $\mathrm{a}=\frac{\mathrm{k}_{\mathrm{H}}}{\mathrm{k}_{\mathrm{v}}}>100$;

- la capacité maximale prévisible d'un puits de rabattement était comprise entre $20 \mathrm{~m}^{3} / \mathrm{h}$ et $50 \mathrm{~m}^{3} / \mathrm{h}$;

- pour rabattre la nappe de $25 \mathrm{~m}$ dans la fouille, il faudrait pouvoir pomper avec un débit moven de $450 \mathrm{~m}^{3} / \mathrm{h}$ à $500 \mathrm{~m}^{3} / \mathrm{h}$.

\section{FOUILLE DE L'USINE}

\subsection{Dimensions}

L'usine est fondée au niveau $(184,50)$ soit à $22,50 \mathrm{~m}$ au-dessous du terrain naturel.

La construction, à l'abri d'une enceinte d'une longueur de $280 \mathrm{~m}$, d'une largeur de $180 \mathrm{~m}$ et d'une profondeur de $22,50 \mathrm{~m}$ environ, a nécessité la réalisation à sec d'une fouille de volume $340000 \mathrm{~m}^{3}$ environ dont $240000 \mathrm{~m}^{3}$ d'alluvions sablo-graveleuses composant le sol de couverture et $100000 \mathrm{~m}^{3}$ environ de sables silteux constituant le fond de la fouille et l'assise de l'usine.

\subsection{Enceinte de protection (fig. 2 et 3 )}

La présence du Rhône à proximité de la fouille de l'usine et la forte perméabilité des alluvions entre le terrain naturel à $(207,00)$ et le niveau de contact des sables silteux à $(195,00)$ ont nécessité la construction d'une coupure étanche des alluvions sablo-graveleuses constituée par une paroi en bentonite-ciment d'épaisseur $62 \mathrm{~cm}$, moulée dans le sol, dont la profondeur déterminée par des conditions de stabilité des talus et du fond de fouilles, a été limitée, après étude sur modèle mathématique, sensiblement au niveau du fond de fouille, soit à la cote $(185,00)$. La paroi a été implantée à $15 \mathrm{~m}$ de la crête des talus périphériques de la fouille.

Le rabattement de la nappe à l'intérieur de l'enceinte délimitée par la paroi étanche a été réalisé, avant le début des terrassements, à l'aide de quarante-cinq puits de diamètre $380 \mathrm{~mm}$ :

- trente-sept puits de $53,50 \mathrm{~m}$ de profondeur, crépinés sur toute leur hauteur, espacés de $20 \mathrm{~m}$, en tête des talus suivant un tracé intérieur et parallèle à celui de la paroi étanche périphérique et disposé à 10 mètres de celle-ci ;

- huit puits de $63,50 \mathrm{~m}$ de profondeur crépinés sur $10 \mathrm{~m}$ à la base.

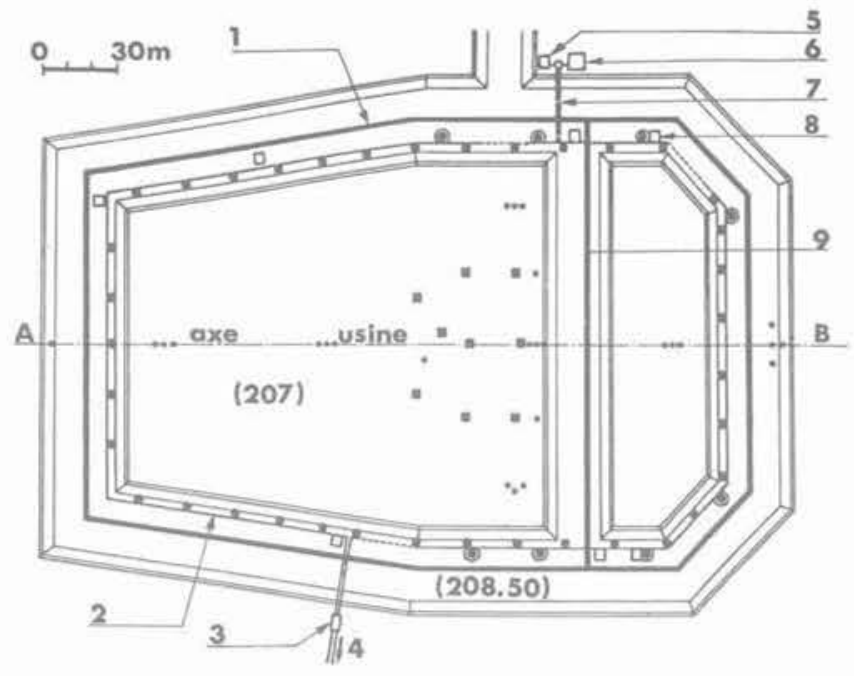

Fig. 2. - Vue en plan (plan view).

\author{
Fig. 2. - Vue en plan \\ 1. Paroi périphérique d'étanchéité - \\ profondeur $27 \mathrm{~m}$ \\ 2. Collecteur en P.V.C. $\varnothing 300 \mathrm{~m}$ \\ 3. Déversoir de mesure \\ 4. Rejet vers le Rhône \\ 5. Groupe électrogène de secours \\ 6. Réseau général d'alimentation électrique \\ 7. Alimentation électrique des pompes \\ 8. Armoires électriques \\ 9. Paroi parafouille - profondeur $40 \mathrm{~m}$
}




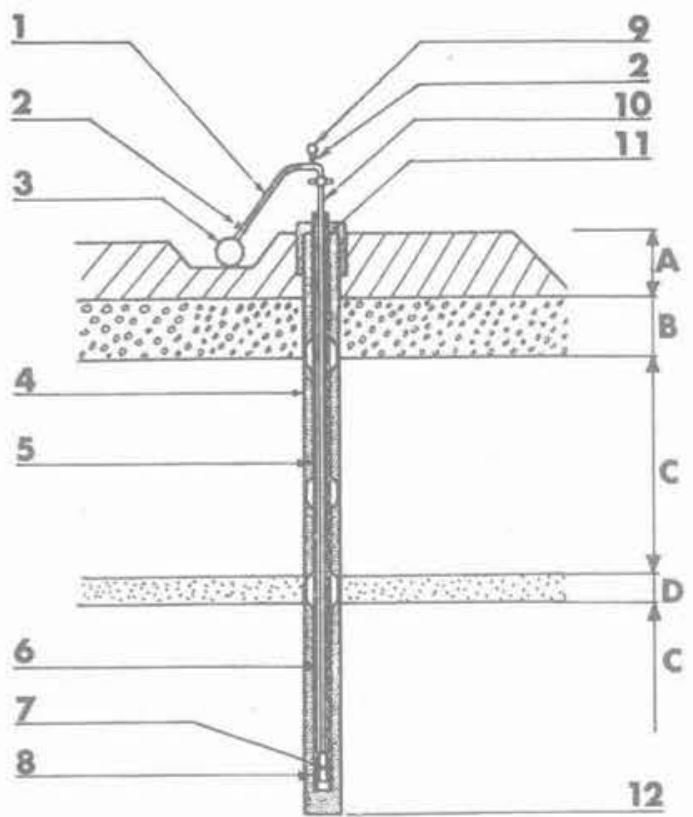

Fig. 3. - Puits de pompage - coupe (filtering well - profile)

1. Tube souple

2. Vanne

3. Collecteur en P.V.C. $\varnothing 300 \mathrm{~mm}$

4. Forage $\varnothing 380 \mathrm{~mm}$

5. Tube métallique crépiné $\varnothing 180 \mathrm{~mm}$

6. Gravier filtre

7. Pompe immergée

8. Tube lisse

9. Manomètre

10. Refoulement $\varnothing 4$

11. Réserve de gravier filtre et protection de tête

12. Fond du forage : niveau (145) ou (155)

A. Terrain rapporté

B. Alluvions graveleuses

C. Sables fins silteux

D. Couches de sables fins silteux plus imperméables

Fig. 3. - Fittrering well - Profile

1. Flexible tube

2. Valve

3. Collecteur P.V.C $\varnothing 300 \mathrm{~mm}$

4. Drilling $\varnothing 380 \mathrm{~mm}$

5. Holed metal tube $\varnothing 180 \mathrm{~mm}$

6. Gravel filter

7. Submerge pump

8. Smoothed metal tube

9. Manometer

10. Hydraulic dredging

11. Filter gravel reserve and top protection

12. Well bottom : nivel (145) or (155)

A. Made ground

B. Gravelly alluvions

C. Silty fine sand

D. Layer of more impervious silty fine sand
Ces puits ont été forés à la boue autodestructible à partir d'une plate-forme de travail à $(208,50)$ ceinturant la foulle constructive lors de la réalisation de la paroi étanche composant l'enceinte de protection.

Les pompes électriques immergées équipant les puits étaient reliées à une tuyauterie collectrice périphérique du rejet avec dispositif de mesure du débit total d'exhaure. Un groupe électrogène thermique assurait le secours automatique de l'alimentation électrique des pompes en cas de défaillance du réseau général E.D.F.

Dans le marché, il était précisé que la piézométrie sous la fondation de l'usine, et principalement au niveau $(165,00)$ N.G.F., serait celle correspondant à une nappe à $(182,00)$ N.G.F. assurant ainsi la stabilité du fond de fouille au soulèvement avec un coefficient de sécurité égal à 2.

Des piézomètres, forés depuis le niveau du terrain naturel suivant la même technique que les puits de rabattement, permettaient de contrôler, à différents niveaux, la cote de rabattement de la nappe fixée à $(182,00)$. Nous noterons que ce niveau n'a pu être atteint qu'après forage de huit puits supplémentaires plus profonds limités à la cote $(145,00)$ environ soit à $39,50 \mathrm{~m}$ environ au-dessous du fond de fouille de P'usine.

Enfin, un ensemble de douze puits de décompression, de mêmes caractéristiques et forés suivant la même technique que les puits de rabattement, assurait la sécurité complémentaire contre le risque de soulèvement du fond de fouille en cas de défaillance du circuit de rabattement.

\subsection{Réalisation de la fouille}

Les travaux de terrassement n'ont été entrepris que lorsque les mesures piézométriques ont permis de contrôler que le niveau de rabattement dans l'emprise de la fouille avait atteint la cote $(182,00)$ environ.

Les terrassements, notamment ceux effectués dans les silts, se sont déroulés dans d'excellentes conditions. Malgré la présence de forages de décompression et piézomètres, recépés jusqu'au niveau du fond de fouille, suivant l'avancement des travaux, les terrassements n'ont été marqués par aucun incident.

Pendant toute la période de rabattement de la nappe à la cote $(182,00)$ le débit horaire moyen pompé s'est stabilisé à $600 \mathrm{~m}^{3}$ environ.

\section{4. Équipement et sécurité du dispositif de rabattement}

\subsubsection{Equipements}

Le dispositif de rabattement comportait :

- trente-sept pompes électriques immergées équipant les puits descendus à la cote N.G.F. (155) et réparties comme suit :

- seize pompes de 10,2 CV

- seize pompes de $5 \mathrm{CV}$ 
- deux pompes de $2.2 \mathrm{CV}$

- trois pompes de $1,5 \mathrm{CV}$;

- huit pompes électriques immergées équipant les puits descendus à la cote N.G.F. (145) et réparties comme suit :

- une pompe de $20 \mathrm{CV}$

- cinq pompes de $17 \mathrm{CV}$

- deux pompes de 10,2 CV

- un collecteur en tube P.V.C. $\varnothing 299 / 315 \mathrm{~mm}$ à double exutoire évacuant les eaux de refoulement des puits de rabattement; ce collecteur, composé de deux boucles symétriques par rapport à l'axe transversal de la fouille, était disposé dans un fossé à quelques mètres de la crête de la fouille et se raccordait, avant rejet dans le Rhône, à un déversoir à lame déversante disposé à l'extérieur de l'enceinte de protection du chantier; le fossé périphérique abritant le collecteur comportait un exutoire vers le Rhône destiné à l'evvacuation des eaux de rabattement en cas de rupture accidentelle du collecteur;

- un groupe électrogène de secours de 275 KVA à démarrage et enclenchement automatique capable d'assurer le fonctionnement des pompes électriques en cas de panne du réseau général électrique de distribution:

- un second groupe électrogène de secours de puissance plus faible, à démarrage manuel, destiné au fonctionnement des pompes prioritaires en cas de panne des réseaux électriques de distribution générale ou de secours.

\subsubsection{Sécurité et maintenance}

La sécurité et la maintenance du dispositif de rabattement ont fait l'objet de soins particuliers :

Étude et analyse détaillées des pannes susceptibles d'affecter le dispositif, élaboration de consignes strictes, désignation d'équipes d'astreinte de surveillance et de dépannage, approvisionnements de matériels et pièces de rechange.

Ces dispositions ont été maintenues jusqu'à ce que la construction de l'usine ait atteint un niveau suffisant compatible avec la stabilité d'ensemble des ouvrages.

\section{5. Épuisements}

Le dispositif de rabattement a fonctionné de façon continue avec satisfaction de juin 1981 à octobre 1983 soit durant vingt-sept mois.

Le volume total de rabattement extrait pendant cette période est de $9500000 \mathrm{~m}^{3}$ environ avec les variations approximatives de débits suivantes :

- Rabattement de (206) à (182) : $300 \mathrm{~m}^{3} / \mathrm{h}$ pendant trois mois.

- Maintien du rabattement à (182) : $580 \mathrm{~m}^{3} / \mathrm{h}$ pendant dix-sept mois.

- Remontée du rabattement de (182) à (196) : $330 \mathrm{~m}^{3} / \mathrm{h}$ pendant sept mois. 